\title{
Long-range symmetry breaking in embedded ferroelectrics
}

Simons, Hugh; Bjørnetun Haugen, Astri; Jakobsen, Anders Clemen; Schmidt, Søren; Stöhr, Frederik; Majkut, Marta; Detlefs, Carsten; Daniels, John E.; Damjanovic, Dragan; Poulsen, Henning Friis

Published in:

Nature Materials

Link to article, DOI:

$10.1038 / \mathrm{s} 41563-018-0116-3$

Publication date:

2018

Document Version

Peer reviewed version

Link back to DTU Orbit

Citation $(A P A)$ :

Simons, H., Bjørnetun Haugen, A., Jakobsen, A. C., Schmidt, S., Stöhr, F., Majkut, M., Detlefs, C., Daniels, J. E., Damjanovic, D., \& Poulsen, H. F. (2018). Long-range symmetry breaking in embedded ferroelectrics. Nature Materials, 17(9), 814-819. https://doi.org/10.1038/s41563-018-0116-3

\section{General rights}

Copyright and moral rights for the publications made accessible in the public portal are retained by the authors and/or other copyright owners and it is a condition of accessing publications that users recognise and abide by the legal requirements associated with these rights.

- Users may download and print one copy of any publication from the public portal for the purpose of private study or research.

- You may not further distribute the material or use it for any profit-making activity or commercial gain

- You may freely distribute the URL identifying the publication in the public portal 


\section{Long-range symmetry breaking in embedded ferroelectrics}

Hugh Simons ${ }^{1 *}$, Astri Bjørnetun Haugen ${ }^{2}$, Anders Clemen Jakobsen ${ }^{1}$, Søren Schmidt ${ }^{1}$, Frederik Stöhr $^{3}$, Marta Majkut ${ }^{4}$, Carsten Detlefs ${ }^{4}$, John E. Daniels ${ }^{5}$, Dragan Damjanovic ${ }^{6}$ and Henning Friis Poulsen ${ }^{1}$

1 Department of Physics, Technical University of Denmark, Fysikvej, Kgs. Lyngby DK-2800, Denmark

2 Department of Energy Conversion and Storage, Technical University of Denmark, Frederiksborgvej 399, Roskilde DK-4000, Denmark

3 DTU Danchip, Technical University of Denmark, Kgs. Lyngby DK-2800, Denmark

4 European Synchrotron Radiation Facility, 71 Avenue des Martyrs, CS40220, 38043 Grenoble Cedex 9, France

5 School of Materials Science and Engineering, UNSW Australia, Kensington, NSW 2052, Australia

6 Group for Ferroelectric and Functional Oxides, Swiss Federal Institute of Technology in Lausanne - EPFL, 1015 Lausanne, Switzerland

*e-mail: husimo@fysik.dtu.dk

The characteristic functionality of ferroelectric materials is due to the symmetry of their crystalline structure. As such, ferroelectrics lend themselves to design approaches that manipulate this structural symmetry by introducing extrinsic strain. Using in-situ dark-field xray microscopy to map lattice distortions around deeply embedded domain walls and grain boundaries in $\mathrm{BaTiO}_{3}$, we reveal that symmetry-breaking strain fields extend up to several micrometers from domain walls. As this exceeds the average domain width, no part of the material is elastically relaxed, and symmetry is universally broken. Such extrinsic strains are pivotal in defining the local properties and self-organization of embedded domain walls, and must be accounted for by emerging computational approaches to material design. 
Extrinsic strains (defined here as any strain in addition to the spontaneous strain) are ubiquitous in ferroelectrics because they are inherent to atomic defects [1], dislocations [2] and internal interfaces such as domain walls [3], grain boundaries [4], and regions connecting epitaxial layers [5]. Since the extrinsic strain tensor is rarely parallel to the spontaneous strain tensor at any given position in the material, their superposition usually reduces local symmetry. This symmetry lowering can be significant: even small distortions of the lattice destabilize the structure in such a way as to facilitate rotation of the polarization [6] which, in some cases can enhance piezoelectric coefficients by up to an order of magnitude [7]. Strain gradients also lead to flexoelectric effects [8] that can give rise to nano-scale photoelectricity and electrical conductivity [9]. More often, however, the uncontrolled presence of defects and their strain fields degrade macroscopic properties by pinning domain walls and suppressing polarisation switching [10]. Whether strain will inhibit or enhance functionality hinges on its magnitude, orientation, symmetry and spatial distribution within the bulk material. Exploiting local strain fields thus requires the ability to spatially resolve and quantify them at the nano-scale within the interior of bulk, macroscopic samples.

\section{Quantitative strain mapping in bulk ferroelectrics}

There are several techniques for mapping strain with high spatial resolution, but stringent sample requirements mean these measurements typically occur under boundary conditions not representative of the bulk material. Transmission electron microscopy (TEM) can resolve atomic displacements [11], but requires sub-100 nanometer-thick samples in which the probed volume is effectively unconstrained in the direction perpendicular to the surface. Similarly, piezoresponse force microscopy [12] and electron back-scatter diffraction microscopy [13] are surface sensitive characterization techniques that do not penetrate the bulk. Comparing strain measurements obtained under different mechanical boundary conditions is inherently problematic because strain fields strongly depend on whether the sampling volume is relaxed or constrained - an issue reflected in the disparate predictions and measurements of domain wall widths made since the 1950s [14]. The lack of bulk sensitive characterization tools is particularly crucial for commercially applicable materials where strain-generating defects and interfaces are typically embedded and constrained by neighbouring grains, domains or clamping effects from substrates or electrodes [15].

Directly measuring the spatial distribution of embedded strain fields requires methods that are penetrating, non-destructive and able to resolve the relevant length scales (i.e. sub- $\mu \mathrm{m}$ ). However, existing scanning-beam x-ray microscopy techniques are slow and therefore limited to surfaces [16], small sampling volumes [17] or reduced resolution [18]. Here, we use dark-field x-ray microscopy [19] - a recent full-field imaging technique for rapidly and quantitatively mapping strain and 
orientation around embedded interfaces. We directly measure the magnitude and distribution of axial strain and lattice misorientation around grain boundaries and ferroelastic domain walls within a buried grain of barium titanate $\left(\mathrm{BaTiO}_{3}\right)$ ceramic, and record in situ the evolution of the embedded domain structure and its local changes in symmetry under electric fields.

\section{Identifying symmetry breaking in a $\mathrm{BaTiO}_{3}$ polycrystal}

$\mathrm{BaTiO}_{3}$ is a prototypical ferroelectric and ferroelastic perovskite with well-known structural and functional properties. It has tetragonal crystal symmetry at room temperature, electrically polarized domains in $<001>$ directions and atomically coherent ferroelastic domain walls along $\{110\}$ planes (i.e. $90^{\circ}$ walls). Hence, diffraction measurements of $\mathrm{BaTiO}_{3}$ 's $\{00 l\}$ lattice planes should result in a reciprocal space map in which the $l 00,0 l 0$ and $00 l$ peaks are distinctly separated by the angle $\gamma=$ $2 \tan ^{-1}(c / a)-90^{\circ} \approx 0.5^{\circ}$, defined by the ratio of the tetragonal lattice parameters, $a$ and $c$. However, our preliminary reciprocal space measurement (i.e. not spatially resolved) of an embedded grain within a $\mathrm{BaTiO}_{3}$ ceramic shows this is not strictly the case (Figure 1). In addition to the three primary peaks (marked $i$ ), the reciprocal space map shows additional complexity, including: $i$ ) additional peaks separated by $\gamma$, iii) additional peaks not separated by $\gamma$, and $i v$ ) diffuse intensity between these peaks. While $i$ ) is evidence of multiple twinning [20], iii) and $i v$ ) indicate local deviations of $\gamma$ that break the nominal tetragonal symmetry. These indicators of broken symmetry have been observed in x-ray reciprocal space maps of ferroelectric films [21] and single crystals [22], where they were attributed to domain walls. Similarly, grain boundaries also locally break symmetry and contribute to the x-ray intensity distribution. However, neither Figure 1 nor previous results provide the spatial distribution of these strain and misorientation fields.
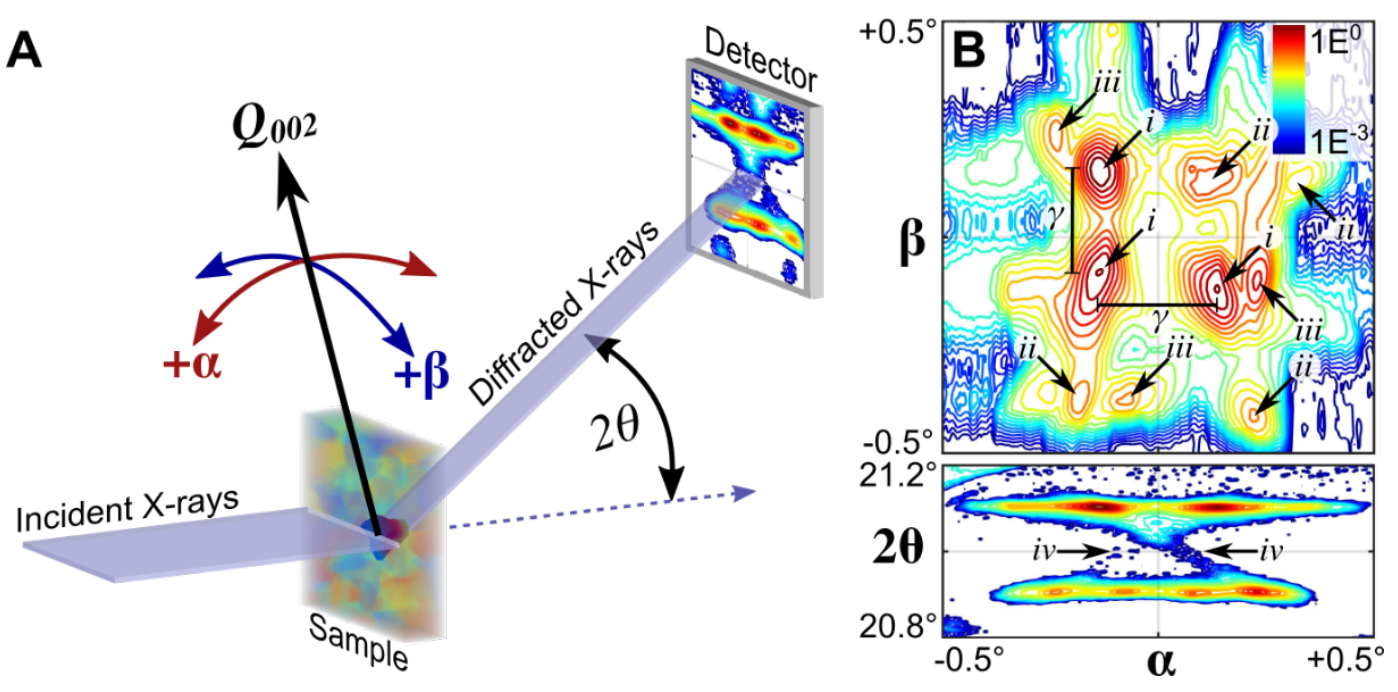

Figure 1. Conventional x-ray diffraction measurement of a single embedded crystallite of BaTiO $_{3}$. In the diffraction geometry (A), the angles $\alpha, \beta$ and $2 \theta$ correspond to the grain tilt around 
the scattering vector, $\boldsymbol{Q}_{002}$ (which for small angles is approximately equal to the misorientation) and scattering angle (see Methods for further details). X-ray integrated intensity maps for the $\alpha-\beta$ and $\alpha-$ $2 \theta$ planes (B) of the 3D intensity distribution (shown in Supplementary Figure 1). Unstrained $90^{\circ}$ walls with the nominal tetragonal symmetry should produce distinct Bragg peaks separated in $\alpha$ and $\beta$ by the angle $\gamma$, marked here by $i$ (primary peaks) and $i i$ (secondary peaks from multiple twinning). The additional peaks and diffuse intensity, marked by iii and $i v$, respectively cannot be accounted for by a consistent tetragonal symmetry.

\section{Mapping strain and structure with dark-field x-ray microscopy}

To establish the topological origin of the symmetry breaking, we recorded a magnified real-space image of the same embedded $\mathrm{BaTiO}_{3}$ grain (Figure 2A) by placing an x-ray objective lens in the Bragg-diffracted beam between the sample and detector. This technique - dark-field x-ray microscopy [19] - is analogous to dark-field transmission electron microscopy, but with the ability to penetrate millimeter-sized samples. When the sample is illuminated with a one-dimensionally focused planar beam, full-field images correspond to a two dimensional "slice" through the embedded grain. In addition to creating the magnified image, passing the diffracted x-rays through the x-ray objective lens also avoids the blurring from strain observed in conventional x-ray section topography and excludes stray diffraction signals from other grains in the sample. Scanning the sample tilt $(\alpha, \beta)$ and scattering $(2 \theta)$ angles, we were able to reconstruct maps of the local $\{002\}$ lattice spacing $\left(\varepsilon_{33}\right.$ strain component) (Figure 2B) and orientation (Figure 2C) with a spatial, orientation and strain resolution of 100-200 nanometers, 1 milliradian and 10 $0^{-5}$, respectively [23]. While only the axial strain is measured directly, the assumption of a coherent lattice implies that the two components of lattice inclination (i.e. $\alpha, \beta$ ) correspond to in-plane gradients of additional strain components. 

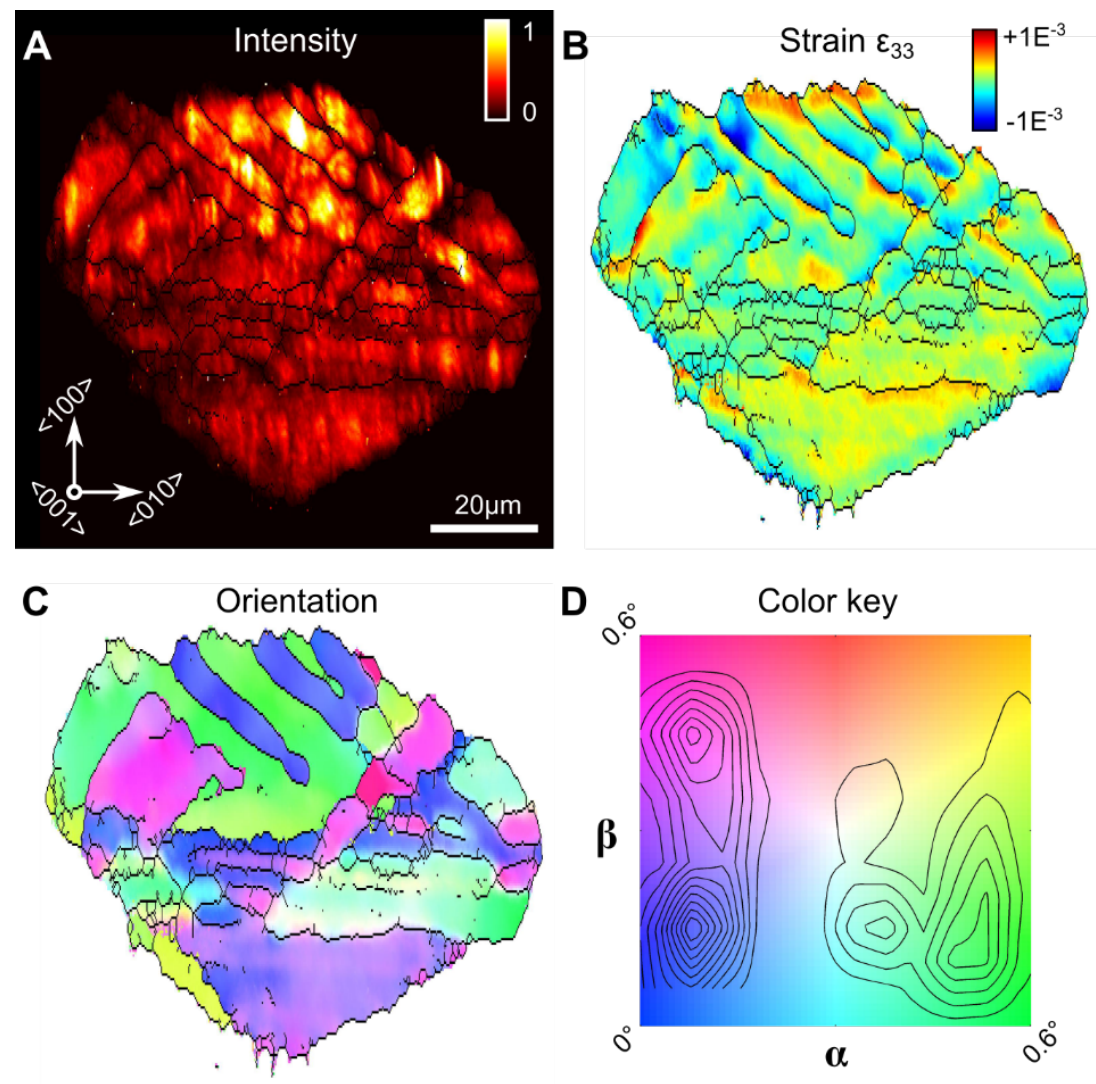

Figure 2. Cross-sectional dark-field x-ray microscopy maps of the embedded $\mathrm{BaTiO}_{3}$ grain. Individual domains are visible in the integrated intensity image (A), while the reconstructed strain (B) and lattice orientation (C) maps reveal the structural relationship between domain clusters. Local orientation $(\alpha, \beta)$ is read from the colour key (D), where the integrated diffraction intensity distribution is superimposed as a contour map. Boundaries (black lines) in (A), (B) and (C) are defined by local orientation gradients exceeding $0.5 \%$ m.

\section{Correlating strain and topology}

The 93 micrometer-wide grain has faceted boundaries surrounded by a strained region approximately 5 micrometres thick. As such, the peripheral region is subject to heterogeneous stress from the grain boundary, whereas the stress in the interior region is widely considered homogeneous [24]. This interior region has a complex domain structure qualitatively consistent with simulations of sheared [25] and isolated volumes [26], and surface measurements of large grains and single crystals [17]. This complex domain structure is also classically hierarchical: Individual micrometer-sized domains are organized in 5-10 micrometer clusters, which in turn have a lamellar arrangement within the grain (Figure 2A). The strain distribution (Figure 2B) is correlated with the domain structure, with larger strain magnitudes associated with $<110>$-oriented domain walls than $<010>$-oriented domain walls. Neighbouring lamellae separated by $<110>$ or $<010>$-oriented domain walls differ in orientation by 
$\sim \gamma\left(0.3-0.5^{\circ}\right)$ (Figure 2C, with colour key in Figure 2D), and are therefore both $90^{\circ}$ domain walls. These strain fields overlap and result in a highly inhomogeneous distribution of strain and strain gradients across the full width of the grain.

The maps contain three types of structural interface, exemplified in Figure 3: in-plane $90^{\circ}$ (where both polarization vectors lie in the observation plane), $<110>$-oriented ferroelastic domain walls characterized by a sharp interface and high-magnitude strain fields extending the full width of the domain $(\sim 5 \mu \mathrm{m})$ (Figure $3 \mathrm{~A})$; out-of-plane $90^{\circ}$ (where at least one polarization vector has a component normal to the observation plane), $<010>$-oriented ferroelastic domain walls characterized by a diffuse interface and lower magnitude strain fields extending a similar distance (Figure 3B); and grain boundaries, which typically have longer-range strain and orientation gradients up to 5-10 $\mu \mathrm{m}$ from the boundary (Figure 3C). Significantly, the domain walls also contain kinks and bends, which are correlated with these strain and orientation gradients.
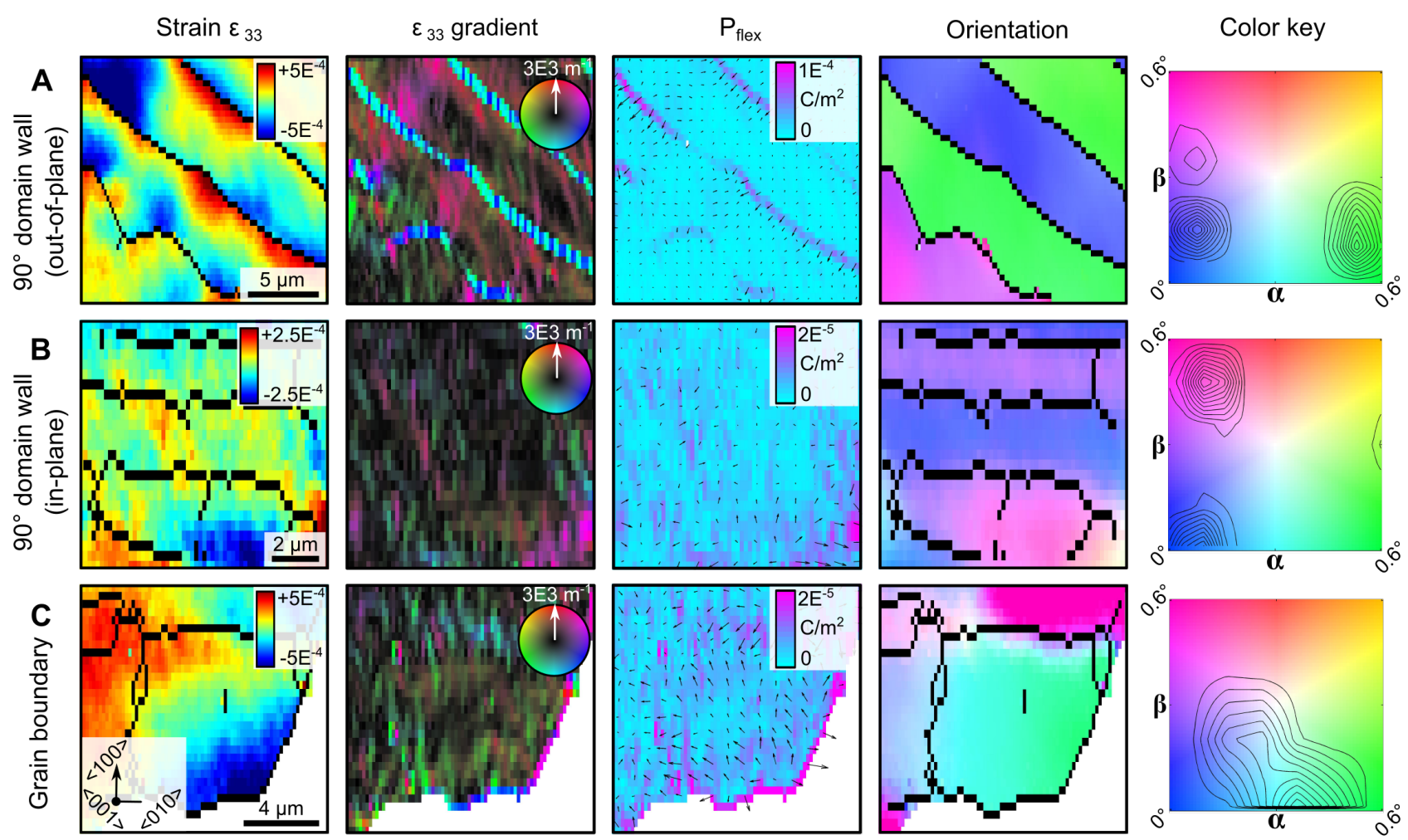

Figure 3. Local lattice distortions around embedded structural interfaces. Quantitative maps of local axial strain, axial strain gradient, flexoelectric polarization, orientation and reciprocal space maps corresponding to $90^{\circ}$ (in-plane) domain walls (A), $90^{\circ}$ (out-of-plane) domain walls (B) and grain boundaries $(\mathrm{C})$. These three types of interfaces have characteristic diffraction intensity distributions closely resembling the additional peaks observed in the Figure 1B-C. 
The widespread correlation between long-range gradients in strain and orientation implies that the local symmetry in the grain is predominantly triclinic - the lowest possible symmetry. Continuous orientation gradients signify a bending strain and an out-of-plane strain gradient. When this overlaps with the observed in-plane strain gradient, the strain state is triaxial. Simulations of complex nanodomain topologies predict that the non-local interaction of triaxial strain fields will result in longrange strain fields with $1 / r^{3}$ decays that extend approximately ten times further than the intrinsic strain fields of the domain walls [25]. Similarly, the strain fields created at the tips of needle domains are also long-ranging and independent of the intrinsic properties of the domain walls [27,28]. However, these predictions are commonly limited to specific topologies and/or sub-micrometer simulation volumes; our measurements suggest that embedded strain fields in bulk materials extend orders of magnitude further, and into the macroscopic regime.

But how do such long-range strain fields influence the piezoelectric and ferroelectric response?

Strain gradients can locally rotate the spontaneous polarization through flexoelectricity [8]. In $\mathrm{PbTiO}_{3}$ thin films, flexoelectrically-induced rotations of the spontaneous polarization of up to several degrees have been measured within a few nanometers of domain walls [21]. In highly-strained $\mathrm{BiFeO}_{3}$ films, flexoelectricity and symmetry lowering were associated with an enhanced piezoresponse around strained nano-sized regions of monoclinic phase in the rhombohedral matrix [29]. In contrast, the gradients observed in Figure 3 have a much smaller magnitude (and thus smaller rotation), but extend an order of magnitude further from domain walls. This does not preclude much larger rotations at length scales below our spatial resolution (such as in [21]). However, the large spatial extent of the strain gradients observed here mean that non-uniform polarization rotations occur statically throughout the entire grain.

Strain also influences local ferroelectric and piezoelectric properties by changing the stability of the ferroelectric phase [6]. When the extrinsic strain is oblique to the spontaneous strain, the symmetry is lowered and the stability of the ferroelectric structure is reduced. Often viewed as a flattening of the free-energy profile, such destabilization is known to permit field-induced rotations of the spontaneous polarization [6]. Thermodynamic calculations predict enhancement of $\mathrm{d}_{33}$ and permittivity by more than an order of magnitude for large electric fields [6], while a ten-fold enhancement of $\mathrm{d}_{33}$ along nonpolar directions has been observed in relaxor ferroelectrics at small electric fields where the structural distortion (presumed to be due to the destabilization of the rhombohedral and tetragonal structures) and ensuing polarization rotations are relatively small [30]. 
Extrinsic strains parallel to the spontaneous strain have the converse affect; stabilizing the ferroelectric structure and reducing piezo- and ferroelectric properties [6]. Therefore, a polycrystalline ceramic containing complex domain patterns will invariably have regions in which properties are enhanced and inhibited. The final macroscopic properties will reflect the ensemble average of these regions. These local properties and phenomena are therefore expected to broadly deviate from ideal behaviour over length scales on the order of the strain fields $(1-5 \mu \mathrm{m})$.

Figures 2 and 3 show clear evidence of pervasive symmetry breaking. A reason this has not been observed previously is that most conventional techniques are not sensitive to local symmetry in bulk samples. For example, powder diffraction measurements of $\mathrm{BaTiO}_{3}$ typically identify the average crystal symmetry at room temperature as tetragonal, not triclinic [31]. However, grain-scale mechanical interactions may locally break the crystal symmetry inferred from the powder-averaged aggregate [32]. Figures 2 and 3 show the topological origin of this anomaly; strain gradients in many directions that, when averaged over many grains within the aggregate, result in isotropic peak broadening and diffuse scattering that obscures the true local symmetry.

\section{Long-range symmetry breaking during electrical poling}

The universal strain inhomogeneity we observe may also affect the bulk switching processes that underlie many ferroelectric applications. Structural distortions at homo-interfaces such as domain walls and grain boundaries can alter domain wall mobility [33] and, in extreme cases, induce correlated switching across clusters of grains [34]. Furthermore, electron microscopy experiments [35] and first principles simulations [36] suggest that point defects preferentially migrate towards strained regions where they potentially become domain wall pinning centres. Orientation maps of the same grain were therefore measured in situ while the electric field was incremented through four critical points of the electric-field-induced ferroelectric response (Figure 4). Importantly, the measurement of a 93 micrometre-wide grain means that the reconfiguration of domains is expected to be observable both inside and outside the heterogeneously stressed region of the grain boundaries. 


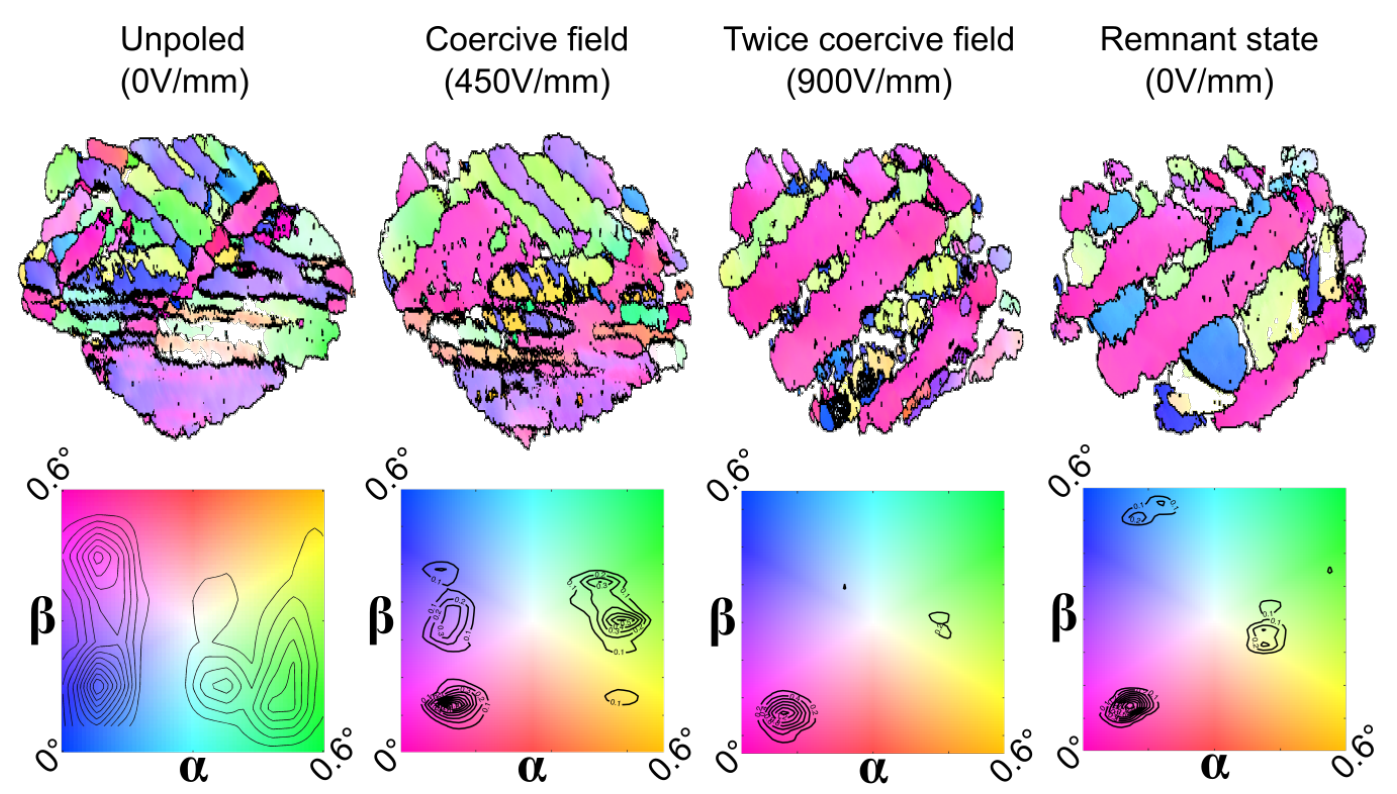

Figure 4. Changes to the domain topology and orientation distribution in the embedded $\mathrm{BaTiO}_{3}$ grain during the in situ application of a unipolar electric field cycle along the $<100>$ direction. Orientation maps (top) and diffraction intensity distributions (bottom) are shown for four points on a characteristic polarization $(P)$ vs. applied electric field $(E)$ hysteresis curve: the initial zero-field state (1); at the coercive field (2) where most domain switching occurs; at twice the coercive field (3) where the induced polarization is saturated; and in the remanent state after the removal of the electric field altogether (4). Videos of the domain evolution as a function of field are available in the Supplementary Materials.

The electric field causes a transformation from a complex domain structure to a classical stripe pattern with a strong preference for $<001>$-oriented domain variants (coloured pink). As expected, wall density decreases, the number of distinct orientations decreases, and domain walls appear mobile (i.e. unpinned) during this poling process. When applying an electric field along a symmetry axis, one would not expect additional symmetry lowering. We observe, however, that the orientation relationships between domains become progressively less consistent with the nominal tetragonal symmetry. Both the orientation intensity distributions and the deviation of the domain wall orientations from their ideal $<110>$ and $<010>$ directions are consistent with local deviation of the domain wall angle $\gamma$, and that the grain-scale average symmetry is lowered.

The poling process favours non-ideal domain orientations despite the increase in strain energy that presumably accompanies them. Since the external field was approximately parallel to the $<001>$ lattice direction of the grain, the unexpected domain pattern must be a consequence of changing grainscale electromechanical boundary conditions during and after poling. The growth of large domains and their piezoelectric and ferroelastic distortions cause increased stresses and depolarization fields 
at grain boundaries. Since these fields are heterogeneous, they induce abnormal domain configurations and widespread symmetry lowering within neighbouring grains. Grain-resolved x-ray diffraction experiments [32] and multi-scale simulations [37] show clear and significant variation in the electromechanical responses of individual grains. Our domain-resolved measurements indicate this variation is due to extrinsic constraints from domains, domain walls and grain boundaries.

\section{Conclusions and outlook}

A clear picture now emerges of how local extrinsic strains affect the ferroelectric topology and response of embedded grains and volumes. We have shown that strained neighbourhoods surrounding embedded grain boundaries and domain walls extend up to several micrometres - approximately an order of magnitude further than many simulations of nano-scale complex domain topologies have predicted [25,27]. These micrometer-range strain fields drastically alter the symmetry of entire grains and, presumably, their ensuing local electromechanical response to applied electric fields. This implies macroscopic behaviour is not strictly defined by the nominal symmetry, but fundamentally affected by local heterogeneities deep within the bulk material. These include widespread networks of strain gradients, which may give rise to local flexoelectric effects. Proposals to utilize domain walls to induce polarization rotation [21] may then be realized using much lower domain wall densities than currently thought.

These results are particularly relevant to multiscale material simulation and design approaches [38]. Instead of systematizing features of the average structure, these approaches should account for the heterogeneous variation of symmetry - and therefore fundamental behaviour - throughout the bulk material. For ferroelastic ceramics, models should simultaneously incorporate nanoscale domain walls, their microscale strain fields, and the effects of their long-ranging symmetry breaking on the macroscopic electromechanical response. This is a major challenge, as the bottom-up approaches needed to accurately model nanoscale phenomena are not feasible at the macroscale. Multiscale modelling approaches [37,38] may be able to reproduce the long-range strain fields we observe, and should be considered in future theoretical work. However, the formulation and validation of such models requires multiscale experimental data, particularly during in-situ and dynamic conditions. The ability to measure quantitative maps of orientation and strain in 3D using dark field x-ray microscopy is a significant advance in this regard, and major improvements in resolution and speed are expected with the unprecedented increase in brilliance and coherence from upcoming fourth generation synchrotron sources [39]. Such multiscale data could be used to directly fit physical parameters for materials models, creating an opportunity to accurately simulate - and ultimately harness - the complex multiscale topologies arising from deeply embedded domains, twins and grain boundaries. 


\section{Methods}

Methods, including statements of data availability and any associated accession codes and references, are available at XXX.

\section{References}

[1] Freedman, D.A., Roundy, D., \& Arias, T.A. Elastic effects of vacancies in strontium titanate: Short- and long-range strain fields, elastic dipole tensors, and chemical strain, Phys. Rev. B 80, 064108 (2009)

[2] Chu, M.-W. , Szafraniak, I., Scholz, R., Harnagea, C., Hesse, D., Alexe M. \& Gösele, U., Impact of misfit dislocations on the polarization instability of epitaxial nanostructured ferroelectric perovskites, Nat. Mater. 3, 87-90 (2004)

[3] Hÿtch, M.J., Snoeck, E. \& Kilaas, R., Quantitative measurement of displacement and strain fields from HREM micrographs, Ultramicroscopy 74, 3, 131-146 (1998)

[4] Choudhury, S., Li, Y.L., Krill III, C. \& Chen, L.-Q., Effect of grain orientation and grain size on ferroelectric domain switching and evolution: Phase field simulations, Acta Mater. 55, 1415-1426 (2007)

[5] Schlom, D.G., Chen, L.-Q., Eom, C.-B., Rabe, K.M., Streiffer, S.K. \& Triscone, J.-M., Strain Tuning of Ferroelectric Thin Films, Annu. Rev. Mater. Res. 37 589-626 (2007)

[6] Budimir, M., Damjanovic, D. \& Setter, N., Enhancement of the piezoelectric response of tetragonal perovskite single crystals by uniaxial stress applied along the polar axis: A freeenergy approach. Phys. Rev. B, 72(6), 064107 (2005)

[7] Noheda, B., Cox, D.E., Shirane, G., Park, S.-E., Cross, L.E. \& Zhong, Z., Polarization rotation via a monoclinic phase in the piezoelectric $92 \% \mathrm{PbZn}_{1 / 3} \mathrm{Nb}_{2 / 3} \mathrm{O}_{3}-8 \% \mathrm{PbTiO}_{3}$, Phys. Rev. Lett., 86, 17, 3891-3894 (2001)

[8] Tagantsev, A.K., Piezoelectricity and flexoelectricity in crystalline dielectrics, Phys. Rev. B, 345883 (1986)

[9] Chu, K., Jang, B.-K., Sung, J.H., Shin, Y.A., Lee, E.-S., Song, K., Lee, J.H., Woo, C.-S., Kim, S.J., Choi, S.-Y., Koo, T. Y., Kim, Y.-H., Oh, S.-H., Jo, M.-H. \& Yang, C.-H., Enhancement of the anisotropic photocurrent in ferroelectric oxides by strain gradients, Nat. Nanotech. 10, 972-979 (2015)

[10] Goncalves-Ferreira, L., Redfern, S.A.T., Artacho, E., Salje, E. \& Lee, W.T., Trapping of oxygen vacancies in the twin walls of perovskite, Phys. Rev. B, 81024109 (2010) 
[11] Chen, C.-C., Zhu, C., White, E.R., Chiu, C.-Y., Scott, M.C., Regan, B.C., Marks, L.D., Huang, Y. \& Miao, J., Three-dimensional imaging of dislocations in a nanoparticle at atomic resolution, Nature 496 74-77 (2013)

[12] Kalinin, S.V. \& Bonnel, D.A., Imaging mechanism of piezoresponse force microscopy of ferroelectric surfaces, Phys. Rev. B 65, 125408 (2002)

[13] Farooq, M.U., Villaurratia, R., Maclaren, I., Kungl, H., Hoffmann, M.J., Fundenberger, J.-J. \& Bouzy, E., Using EBSD and TEM-Kikuchi patterns to study local crystallography at the domain boundaries of lead zirconate titanate, J. Microsc. 320, 445-454 (2008)

[14] Tagantsev, A.K., Cross, L.E. \& Fousek, J., Domains in ferroic crystals and thin films, Spinger, New York (2010)

[15] Uchino, K ., Ferroelectric Devices, CRC Press, (2009)

[16] Hruszkewycz, S.O., Highland, M.J., Holt, M.V., Kim, D., Folkman, C.M., Thompson, C., Tripathi, A., Stephenson, G.B., Hong, S. \& Fuoss, P.H., Imaging local polarization in ferroelectric thin films by coherent x-ray Bragg projection ptychography, Phys. Rev. Lett. 110, $177601(2013)$

[17] Lummen, T.T.A., Gu, Y., Wang, J., Lei, S., Xue, F., Kumar, A., Barnes, A. T., Barnes, E., Senev, S., Belianinov, A., Holt, M., Morozovska, A. N., Kalinin, S. V., Chen, L.-Q. \& Gopalan, V., Thermotropic phase boundaries in classic ferroelectrics, Nat. Commun. 5, 3172, (2014)

[18] Rogan, R.C., Tamura, N., Swift, G.A. \& Üstündag, E., Direct measurement of triaxial strain fields around ferroelectric domains using x-ray microdiffraction, Nat. Mater. 2, 379-381 (2003)

[19] Simons, H., King, A., Ludwig, W., Detlefs, C., Pantleon, W., Schmidt, S., Stöhr, F., Snigireva, I., Snigirev, A. \& Poulsen, H.F., Dark -field x-ray microscopy for multiscale structural characterization, Nat. Commun. 6, 6098 (2015)

[20] Cayron, C., Quantification of multiple twinning in face centred cubic materials, Acta Mater. 559, 1, 252-262 (2011)

[21] Catalan, G., Lubk, A., Vlooswijk, A.H.G., Snoeck, E., Magen, C., Janssens, A., Rispens, G., Rjinders, G., Blank, D.H.A. \& Noheda, B., Flexoelectric rotation of polarization in ferroelectric thin films, Nat. Mater. 10, 963-967 (2011)

[22] Locherer, K.R., Chrosch, J. \& Salje, E.K.H., Diffuse X-ray scattering in $\mathrm{WO}_{3}$, Phase Transitions 67(1), 51-63, (1998)

[23] Simons, H., Jakobsen, A.C., Ahl, S.R., Detlefs, C. \& Poulsen, H.F., Multiscale 3D characterization with dark-field x-ray microscopy, MRS Bulletin 41 454-459 (2016) 
[24] Arlt, G., The influence of microstructure on the properties of ferroelectric ceramics, Ferroelectrics 104(1), 217-227, (1990)

[25] Salje, E.K.H., Li, S., Stengel, M., Gumpsch, P., Ding, X., Flexoelectricity and the polarity of complex ferroelastic twin patterns, Phys. Rev. B 94(2), 024114, (2016)

[26] Ahluwalia, R., Ng, N., Schilling, A., McQuaid, R.G.P., Evans, D.M., Gregg, J.M., Srolovitz, D.J. \& Scott J.F., Manipulating ferroelectric domains in nanostructures under electron beams, Phys. Rev. Lett. 111(16), 165702, (2013)

[27] Novak, J., Bismayer, U., Salje, E.K.H., Simulated equilibrium shapes of ferroelastic needle domains, J. Phys. Condens. Matter. 14(3), 657 (2002)

[28] Harrison, R.J. \& Salje, E.K.H., Ferroic switching, avalanches, and the Larkin length: Needle domains in $\mathrm{LaAlO}_{3}$, Appl. Phys. Lett. 99(15) 151915 (2011)

[29] Cheng, C.E., Liu, H.J., Dinelli, F., Chen, Y.C., Chang, C.S., Chien, F.S.S. \& Chu, Y.H., Revealing the flexoelectricity in the mixed-phase regions of epitaxial $\mathrm{BiFeO}_{3}$ thin films. Sci. Rep. 58091 (2005)

[30] Zhang, R., Jiang, B. \& Cao, W., Orientation dependence of piezoelectric properties of single domain $0.67\left(\mathrm{Mg}_{1 / 3} \mathrm{Nb}_{2 / 3}\right) \mathrm{O}_{3}-0.33 \mathrm{PbTiO}_{3}$ crystals, Appl. Phys. Lett., 82, 3737-3739, (2003)

[31] Kwei, G.H., Lawson, A.C., Billinge, S.J.L. \& Cheong, S.W., Structures of the ferroelectric phases of barium titanate. J. Phys. Chem., 97(10), 2368-2377 (1993)

[32] Daniels, J.E., Majkut, M., Cao, Q., Schmidt, S., Wright, J., Jo, W. \& Oddershede, J., Heterogeneous grain-scale response in ferroic polycrystals under electric field, Sci. Rep. 6 , 22820 (2016)

[33] Marincel, D.M., Zhang, H., Kumar, A., Jesse, S., Kalinin, S.V., Rainforth, W.M., Reaney, I.M., Randall, C.A. \& Trolier-McKinstry, S., Influence of a single grain boundary on domain wall motion in ferroelectrics. Adv. Funct. Mater., 24(10), 1409-1417 (2014)

[34] Bintachitt, P., Trolier-McKinstry, S., Seal, K., Jesse, S. \& Kalinin, S.V., Switching spectroscopy piezoresponse force microscopy of polycrystalline capacitor structures. Appl. Phys. Lett., 94(4), 042906 (2009)

[35] Rojac, T., Bencan, A., Drazic, G., Sakamoto, N., Ursic, H., Jancar, B., Tavcar, G., Makarovic, M., Walker, J., Malic, B. \& Damjanovic, D., Domain-wall conduction in ferroelectric $\mathrm{BiFeO}_{3}$ controlled by accumulation of charged defects, Nat. Mater. 16(3), 322327 (2017)

[36] Aschauer, U., Pfenniger, R., Selbach, S.M., Grande, T. \& Spaldin, N., Strain-controlled oxygen vacancy formation and ordering in $\mathrm{CaMnO}_{3}$, Phys. Rev. B 88, 05411 (2013)

[37] Daniel, L., Hall, D.A. \& Withers, P.J., A multiscale model for reversible ferroelectric behavior of polycrystalline ceramics, J. Mech. Mater. 71, 85-100 (2014) 
[38] Horstmeyer, M.F., Integrated Computational Materials Engineering (ICME) for Metals:

Using Multiscale Modeling to Invigorate Engineering Design with Science, Wiley (2012)

[39] Eriksson, M., van der Veen, J.F. \& Quitmann, C., Diffraction-limited storage rings, J, Synchrotron Radiat., 21, 837-842 (2014)

\section{Additional information}

Supplementary information is available for this paper at XXX

Reprints and permissions information is available at XXX

Correspondence and requests for materials should be addressed to H.S.

\section{Acknowledgements}

We are grateful to the European Synchrotron for providing beamtime at ID06, and Danscatt for financial assistance related thereto. This work is supported by the ERC Advanced grant 'd-TXM' (291321). In addition, H.S. and A.B.H. are supported by individual postdoc grants from the Independent Research Fund Denmark (DFF - 4093-00296 and DFF - 6111-00440). The work of D.D. has been supported by the Swiss National Science Foundation (grant No. 200021-159603), while J.E.D. acknowledges financial support from the ARC Discovery Projects DP120103968 \& DP130100415. The silicon compound refractive lenses used in the experiment where manufactured at DTU Danchip, National Center for Micro -and Nanofabrication.

\section{Author contributions}

A.B.H. and H.S. prepared the samples. H.S., A.C.J., C.D. and M.M. performed the experiments. F.S. developed the x-ray optics for the experiment. S.S. developed the noise-reduction algorithm used in the analysis. H.S. designed the experiment with D.D. and J.E.D. H.S. performed the analysis, then interpreted the data with D.D., J.E.D. and H.F.P. H.S. wrote the article and all authors contributed and commented on the text.

\section{Competing interests}

The authors declare no competing interests. 


\section{Methods}

Sample preparation. $\mathrm{BaTiO}_{3}$ ceramic pellets were prepared by sintering $\mathrm{BaTiO}_{3}$ powder $(99.5 \%$, > $2 \mu \mathrm{m}$ particle size, Sigma Aldrich) at $1300{ }^{\circ} \mathrm{C}$ for 20 hours. The density was $99 \%$ of theoretical and the average grain size was $100 \mu \mathrm{m}$. Rectangular plates were cut from these pellets and mechanically polished to final dimensions of $10 \times 4 \times 0.16 \mathrm{~mm}^{3}$. After polishing, they were annealed at $400{ }^{\circ} \mathrm{C}$ and air-cooled to ambient temperature. Electrodes of silver paste (RS Components) were painted on both $10 \times 4 \mathrm{~mm}^{2}$ surfaces. The sample was then glued to the sample stage and copper electrical connections using the same silver paste.

X-ray diffraction measurements. All measurements were carried out at the hard x-ray microscope at ID06 of the European Synchrotron Radiation Facility (ESRF). An x-ray energy of $17 \mathrm{keV}$ was used, with a bandwidth of $10^{-5}$ (rms) selected by a Si 111 double monochromator. A Be transfocator [40] $20 \mathrm{~m}$ from the undulator pre-focused the x-rays into a Si compound refractive lens [41] located $38 \mathrm{~m}$ downstream. The Si lens focused in the vertical direction only with a focal length of $22 \mathrm{~cm}$. The beam profile at the focal point was approximately Gaussian, with dimensions of $200 \mu \mathrm{m}$ (horizontal) $\times 200 \mathrm{~nm}$ (vertical), confirmed by knife scanning. The sample and its environment were positioned at this focal point such that the beam entered the sample at the approximate centre of the $10 \times 4 \mathrm{~mm}^{2}$ face connected to the positive electrical terminal. At this position, a grain was located with a $\{200\}$ scattering vector approximately perpendicular (within $5^{\circ}$ ) to both the electric field and the horizontal plane. CCD detectors were then positioned to image the $\{200\}$ Bragg peak at a distance of $5.5 \mathrm{~m}$ from the sample. The sample geometry is identical to the diffraction geometry given in Figure 1A of the main text, albeit with the x-ray objective lens placed in the diffracted beam path, approximately $25 \mathrm{~cm}$ from the sample. A detailed schematic of this geometry is also given in Simons et al. [19].

Reciprocal space maps. The reciprocal space maps used a Basler CCD camera and the optics necessary to produce a circular $50 \mathrm{~mm}$ field of view with $54 \mu \mathrm{m}$ pixels. This field of view sampled approximately $2.22^{\circ}$ in $\beta$ and $0.55^{\circ}$ in $2 \theta$ in each image. Exposures of 1 second were recorded while incrementally tilting the $\alpha$ angle through $0.6^{\circ}$ in 500 steps. These image series were then digitally interpolated into linear $\alpha-\beta-2 \theta$ space.

Dark-field $x$-ray microscopy. The dark-field $\mathrm{x}$-ray microscopy measurements required inserting an $\mathrm{x}$ ray objective lens and a higher-resolution CCD camera into the diffracted beam path. The x-ray objective comprised a Be compound refractive lens with a focal length of $25 \mathrm{~cm}$, which gave an $\mathrm{x}$ ray magnification of $15.9 \times$ at a numerical aperture of $5 \times 10^{-4}$. The FReLoN CCD camera used a LAG scintillator and a $10 \times$ microscope objective yielding an effective pixel size of $1.4 \mu \mathrm{m}$. Combined, the 
dark-field microscope had a spatial resolution of approximately $100 \mathrm{~nm}$ (horizontal) $\times 200 \mathrm{~nm}$ (vertical) $\times 200 \mathrm{~nm}($ depth). Image acquisition consisted of recording a 3 second exposure as the sample was progressively tilted through $\alpha$ and $\beta$, and the objective and detector tilted through $2 \theta$ in 20 steps over $1^{\circ}$ per direction.

In-situ electrical poling. Constant-magnitude electric fields were applied in-situ during the diffraction and microscopy measurements using a DC power source. First, x-ray reciprocal space maps and darkfield x-ray microscopy maps (both orientation and strain) were recorded without an applied field (i.e. while the sample was in its as-processed state, c.f. Map \#1 in Figure 4A). Next, the electric field magnitude was increased from 0 to $472 \mathrm{~V} / \mathrm{mm}(72 \mathrm{~V})$ over $\sim 12$ minutes. To capture the topological reconfiguration during this increase, dark-field x-ray microscopy images were recorded along with the instantaneous current and voltage (see Supplementary Video S1). After leaving the sample at this electric field for an hour to allow the domain structure to equilibrate, $\mathrm{x}$-ray reciprocal space maps and dark-field x-ray microscopy maps of orientation and strain were then measured (c.f. Map \#2 in Figure 4A). The applied electric field was then increased again from 472 to $944 \mathrm{~V} / \mathrm{mm}(144 \mathrm{~V})$ over $\sim 12$ minutes while simultaneously recording the topological reconfiguration (see Supplementary Video S2). The sample was again allowed to equilibrate at this electric field magnitude before measuring $x-$ ray reciprocal space maps and dark-field x-ray microscopy maps of orientation and strain (c.f. Map \#3 in Figure 4A). Finally, the applied electric field was reduced from 944 to $0 \mathrm{~V} / \mathrm{mm}$ while continuously recording images (see Supplementary Video S3). After equilibration, final x-ray reciprocal space maps and dark-field x-ray microscopy maps of orientation and strain (c.f. Map \#4 in Figure 4A) were measured.

Data reconstruction. All images were pre-processed using a rotational noise-reducing filter. The reciprocal space position $(\alpha, \beta, 2 \theta)$ of each $(\mathrm{x}, \mathrm{y})$ pixel in the image series was then determined by finding the $3 \mathrm{D}$ centroid of the intensity distribution. Domain and grain boundaries were identified based on local lattice orientation gradients, and the local lattice strain was calculated relative to the centroid position of the spatially- and orientation-integrated $2 \theta$-intensity distribution. We note here that our use of the word "orientation" in this work is appropriate only when the tilt angles $\alpha$ and $\beta$ are small. These angles in fact represent the direction of the scattering vector relative to a nominal direction, and only when these angles are small do they approximately correspond to the real misorientation of the lattice. Furthermore, we note that true lattice orientation requires three orientation angles to describe uniquely. Here, we quantify only two of these ( $\alpha$ and $\beta$ ), while the third angle (around the scattering vector, normal to the diffracting planes) remains unresolved. 
For a complete description of dark-field x-ray microscopy and the associated reconstruction techniques, we refer the reader to Simons et al. [19] and Poulsen et al. [42].

Calculation of flexoelectric effect. The flexoelectric effect describes the linear coupling between electrical polarization and strain gradient according to equation 1:

$P_{l}=\mu_{i j k l} \frac{\partial \varepsilon_{i j}}{\partial x_{k}}$

Where $P_{l}$ is the flexoelectric contribution to the polarization, $\mu_{i j k l}$ is the flexoelectric tensor, $\varepsilon_{i j}$ is the strain tensor and $x_{k}$ gives the direction of the strain gradient.

The dark-field x-ray microscopy measurements provide direct maps of $\varepsilon_{33}$ (i.e. out-of-plane strain) from which the strain gradients $\frac{\partial \varepsilon_{33}}{\partial x_{1}}$ and $\frac{\partial \varepsilon_{33}}{\partial x_{2}}$ can be calculated. Maps of these strain gradient components, as well as the strain gradient magnitude $\left(\bar{x}=\sqrt{x_{1}^{2}+x_{1}^{2}}\right)$ for the as-processed (i.e. no applied electric field) state are shown in Supplementary Figure S2 A-C. A histogram of the strain gradient magnitude then reveals the statistical distribution of strain gradients throughout the measured volume (Supplementary Figure S2 D).

The mean and median strain gradients are $1.2 \mathrm{~mm}^{-1}$ and $0.8 \mathrm{~mm}^{-1}$, respectively, while the theoretically derived value (i.e. using DFT) of the flexoelectric coefficient is $\mu_{12}=-5.463 \mathrm{nC} / \mathrm{m}$ [43]. We then use Equation $\mathrm{S} 1$ to estimate the mean flexoelectric polarization contribution as being $P_{\text {flexo }}=5.463 \mathrm{nC} / \mathrm{m}$ $\times 1.2 \mathrm{~mm}^{-1}=6.56 \mu \mathrm{C} / \mathrm{m}^{2}$. The spontaneous (i.e. intrinsic) polarization for $\mathrm{BaTiO}_{3}, P_{s}$, is $\sim 0.26 \mathrm{C} / \mathrm{m}^{2}$. Since the measured strain is out-of-plane, the calculated strain gradients and the ensuing flexoelectric polarization contribution will be in-plane. If such an in-plane polarization contributes to an out-ofplane spontaneous polarization, then the mean polarization rotation angle due to in-plane flexoelectricity can be approximated by $\varphi_{P}=\arctan \left(6.56 \mathrm{E}^{-6} / 0.26\right)=0.0014^{\circ}$. The same approach is used to calculate the maximum effect in more highly strained regions e.g. near grain boundaries and domain walls.

Data availability. All data, in addition to that shown in the Supplementary Information, are available from the authors by request.

\section{References}


[40] Vaughn, G.B.M., Wright, J.P., Bytchkov, A., Rossat, M., Gleyzolle, H., Snigireva, I. \& Snigirev, A., X-ray transfocators: focusing devices based on compound refractive lenses, $J$. Synchrotron Rad. 18 125-133 (2011)

[41] Stöhr, F., Michael-Lindhard, J., Hübner, J., Jensen, F., Simons, H., Jakobsen, A.C., Poulsen, H.F. \& Hansen, O., Sacrificial structures for deep reactive ion etching of high-aspect ratio kinoform silicon x-ray lenses, J. Vac. Sci. Technol. B 33(6) 062001 (2015)

[42] Poulsen, H.F., Jakobsen, A.C., Simons, H., Ahl, S.R., Cook, P.K. \& Detlefs, C., X-ray diffraction microscopy based on refractive optics, J. Appl. Cryst. 50(5), 1441-1456 (2017)

[43] Maranganti, R. \& Sharma, P., Atomistic determination of flexoelectric properties of crystalline dielectrics, Phys. Rev. B. 80, 054109 (2009) 